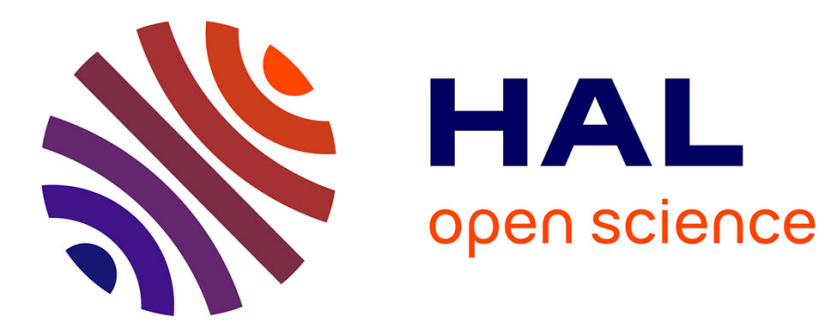

\title{
Stochastic Analysis of Scattered Field by Building Facades Using Polynomial Chaos
}

\author{
P. Kersaudy, S. Mostarshedi, B. Sudret, O. Picon, J. Wiart
}

\section{To cite this version:}

P. Kersaudy, S. Mostarshedi, B. Sudret, O. Picon, J. Wiart. Stochastic Analysis of Scattered Field by Building Facades Using Polynomial Chaos. IEEE Transactions on Antennas and Propagation, 2014, 62, pp.6382 - 6393. 10.1109/TAP.2014.2359478 . hal-01432519

\section{HAL Id: hal-01432519 https://hal.science/hal-01432519}

Submitted on 11 Jan 2017

HAL is a multi-disciplinary open access archive for the deposit and dissemination of scientific research documents, whether they are published or not. The documents may come from teaching and research institutions in France or abroad, or from public or private research centers.
L'archive ouverte pluridisciplinaire HAL, est destinée au dépôt et à la diffusion de documents scientifiques de niveau recherche, publiés ou non, émanant des établissements d'enseignement et de recherche français ou étrangers, des laboratoires publics ou privés. 


\section{GHzürich}

\section{StOCHASTIC ANALYSIS OF SCATTERED FIELD by BUILDING FACADES USING POLYNOMIAL CHAOS}

P. Kersaudy, S. Mostarshedi, B. Sudret, O. Picon, and J. Wiart

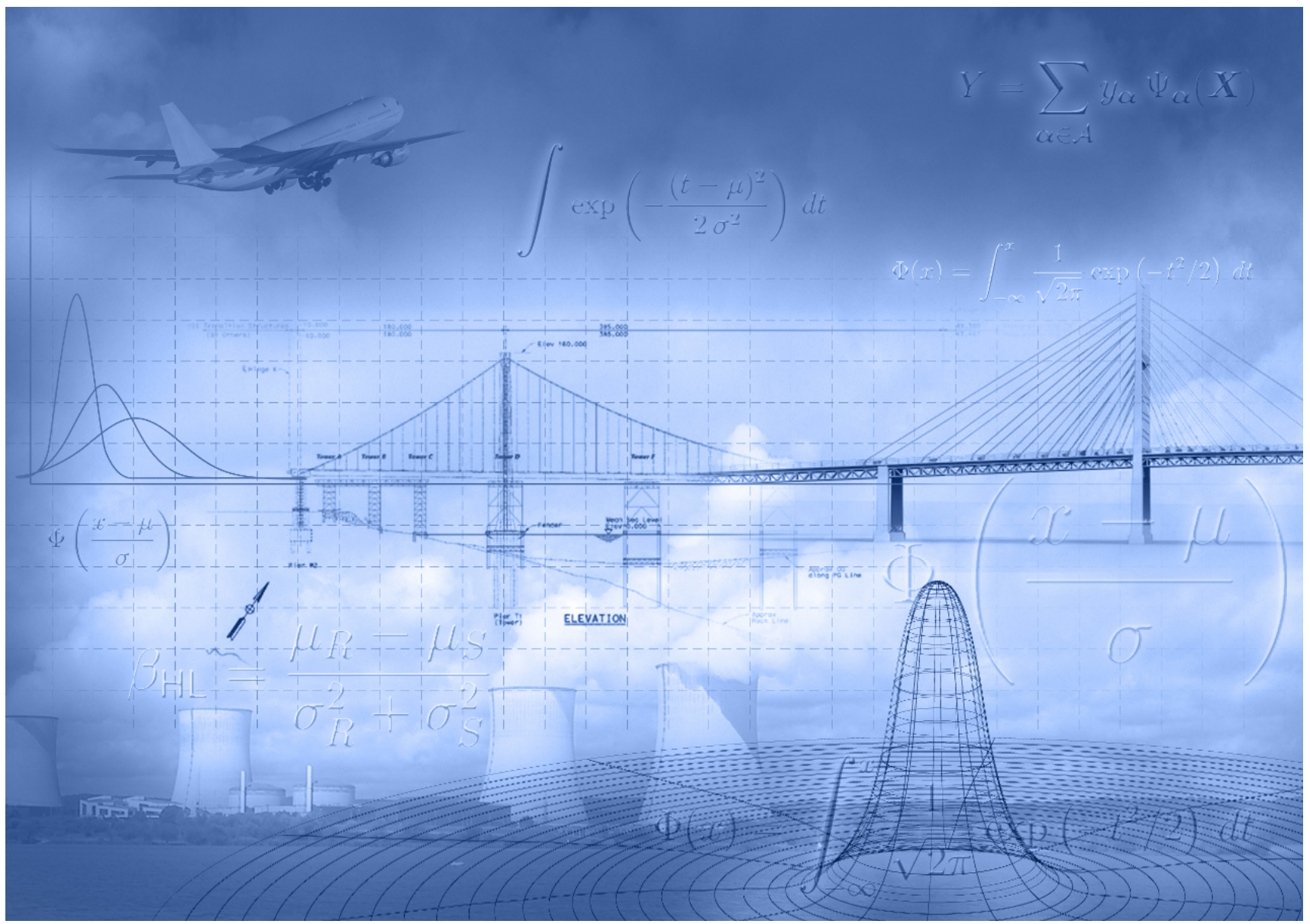




\section{Data Sheet}

ETH Project:

\section{Project ID:}

Journal:

IEEE Trans. Antennas Propagation

Report Ref.:

RSUQ-2014-004

Arxiv Ref.:

DOI:

10.1109/TAP.2014.2359478

Date submitted: July 2014

Date accepted: December 2014 


\title{
Stochastic Analysis of Scattered Field by Building Facades Using Polynomial Chaos
}

\author{
P. Kersaudy, S. Mostarshedi, B. Sudret, O. Picon, and J. Wiart
}

\begin{abstract}
This paper presents a statistical assessment of scattered field from a building facade having random physical and geometrical parameters. A simple inhomogeneous model is considered for the building and the calculation method is based on Green's functions. The basis of polynomial chaos expansion method is explained and applied to estimate the scattered electric field from a building facade having 8 random parameters, in specular and non-specular scenarios. Uncertainty analysis and total output distribution are discussed in different diffraction zones of the building.
\end{abstract}

Index Terms-Scattering, urban environment, architectural details, uncertainty analysis, polynomial chaos

\section{INTRODUCTION}

$\mathrm{W}$ IRELESS communications, particularly mobile networks, experienced a great expansion worldwide. These networks are very dense in urban areas and the electromagnetic field distribution is highly dependent on city structures. It is essential to have predictive tools to assess as accurately as possible the distribution of electromagnetic fields in order to enable optimized implementation of base stations and respect the constraints regarding human exposure. Wave propagation simulators are based on different models of urban environment with different assumptions on the building architectural details. Given the adopted model, they incorporate appropriate calculation methods. We can mention empirical, statistical, theoretical, site-specific models or a combination of them to generate a hybrid model [1]. Empirical models [2] are based on the extensive measurement campaigns and are highly related to the structure of the city. Statistical models [3] are also based on measurements and are used to give wide band multipath model of the environment. Theoretical (physical) [4] models are obtained by imposing some general idealized conditions on the geometry. They usually use physical optics with or without diffraction phenomena. Site-specific models [5] depend on a large number of parameters which are related to a given site and their computing time, depending on the complexity of the environment, can be long. These models usually employ ray techniques, such as ray tracing and ray launching. Given the

Manuscript received March, 2014, revised July 2014.

P. Kersaudy and J. Wiart are with Orange Labs, 80305 Issy-lesMoulineaux, France.

S. Mostarshedi and O. Picon are with ESYCOM (EA 2552), Université Paris-Est, 77524 Marne-la-Vallée, Fance. (phone: +33160957313; fax: +33160957557; e-mail: shermila.mostarshedi@ univ-mlv.fr).

B. Sudret is with the Institute of Structural Mechanics, ETH, CH-8093 Zurich, Swiss. richness of digital map databases nowadays, site-specific models are widely used in wave propagation simulators. Prediction of electromagnetic field intensity in a complex and variable environment is meaningful when presented with an acceptable amount of uncertainty. Yet this last commonly used category of simulators does not accept variable parameters for building architectural details and seldom reports extra information concerning the uncertainty of the results. Therefore, there is a real need to assess the electromagnetic waves in urban environment in terms of the propagation of uncertainties.

Different numerical methods allow the incorporation of the stochastic dimension of the problem to the simulations and measurements. Without being exhaustive, we can mention Monte Carlo method, perturbation techniques, moment equations, operator based methods, polynomial chaos, etc [6], [7]. The final goal of all these techniques is to provide more reliable results concerning a complex system while the incomplete knowledge of the underlying physics and/or inevitable measurement errors naturally generate some imprecision in the prediction of the system's model or simulator. Many domains such as civil engineering, fluid mechanics, control, etc., dealing with large-scale complex systems, have made use of numerical methods for stochastic treatments. More specifically in the electromagnetic field and wave propagation domain, some recent studies have shown interest in different aspects of uncertainty management. Stochastic collocation has been used in radar problems [8], human exposure estimation [9] and reverberation chambers [10]. Polynomial chaos expansion has been introduced for computational electromagnetics [11] and recently been used in EMC problems, for human exposure estimation [12] and cross talk assessment [13]. To our knowledge, no comprehensive study has focused on the propagation of uncertainties related to building geometrical and physical properties. However, these variable parameters may cause non negligible errors in the field estimation in the vicinity of buildings if they are all treated as simple homogeneous blocks.

In a recent publication [14], we applied the "perturbation technique" to the scattered field from a building facade. The perturbation method [7] is based on the Taylor series expansion of the response function around the mean value of the input parameters. The approximation remains valid if the variations of the input parameters around their mean are small. If the input variables are independent, the variance can be decomposed into the contribution of each single input parameter. The sensitivity indices can be calculated using each 
input variance, the total output variance and the first order derivative of the output function with respect to each input parameter. It is important to note that this technique is completely deterministic and needs no sampling and no important computational time. The only mathematical operation is a numerical estimation of the derivative of the function. However the first order expansion cannot always describe the behavior of the model. In the case of the reflected electric field from a building, this technique is no longer valid in the very near-field region where the variation of the electric field around its mean is important. The technique in its validity domain (beyond the very-near-field region) can only offer the simple sensitivity indices and cannot provide the total output distribution.

In the same publication, the results are compared to Monte Carlo simulation [15] which is a more universal approach to estimate the output distribution but may require substantial computer resources. A probability density function is assigned to each input parameter according to which they are sampled. Moreover, from the law of large numbers, the experiment is repeated a considerable number of times in order to obtain a large confidence in the output values. Despite the simple implementation of this non-intrusive method, the convergence rate of the statistical moments such as the mean and the variance is rather low $(1 / \sqrt{N})$. For the scattering problem of interest, this method can potentially give viable results even in the very near-field zone of the building but the required large number of simulations limits its applicability.

In this paper, we treat the problem of variation of the scattered field from a building presenting several random physical and geometrical parameters. For the sake of calculation efficiency, we apply polynomial chaos expansion. In section II, the constitutive equations and associated computational model is presented and the random parameters are introduced. In section III, the basis of polynomial chaos expansions is explained. The series truncation, the method convergence and the quality of the approximation are discussed. Consequently, the sensitivity indices are introduced and interpreted. Finally, some numerical results for two different scattering scenarios are presented in section IV.

\section{SimUlATOR}

\section{A. Method}

The use of a non intrusive technique for the stochastic processing allows a flexible choice of the calculating core of the simulator. Given our other publications, in this paper we continue to utilize the asymptotic method based on the Green's functions [16]; however any other asymptotic, analytical or numerical method commonly employed in wave propagation processing can be used instead (e.g. physical optics, ray tracing, FDTD, etc).

According to this method, a locally plane wave illuminating an object can be replaced, with respect to the induction theorem follows:

$\overrightarrow{J_{s}}=-\hat{n} \times \overrightarrow{H_{i}}$
$\overrightarrow{M_{s}}=\hat{n} \times \overrightarrow{E_{i}}$

where $\hat{n}$ is the, by electric $\left(\overrightarrow{J_{s}}\right)$ and magnetic $\left(\overrightarrow{M_{s}}\right)$ surface currents at the interface between the air and the object. The expressions of the equivalent currents depending only on the incident field are as

local surface normal vector of the object. The total radiation of these fictive currents in the presence of the object gives the scattered field from the object. In order to calculate the radiation of surface currents, the electric $\left(\overline{\bar{G}}_{E J}\right)$ and magnetic ( $\left.\overline{\bar{G}}_{E M}\right)$ Green's functions associated with the interface between two semi-infinite media are used. The convolution integral of the Green's functions with the surface currents gives the reflected field at each point in space:

$$
\vec{E}=\iint_{s^{\prime}} \overline{\bar{G}}_{E J}\left(r, r^{\prime}\right) \bullet \vec{J}_{S}\left(r^{\prime}\right) \overrightarrow{d s^{\prime}}+\iint_{s^{\prime}} \overline{\bar{G}}_{E M}\left(r, r^{\prime}\right) \bullet \vec{M}_{S}\left(r^{\prime}\right) \overrightarrow{d s^{\prime}}
$$

The detailed expressions and explanation on the difference between this type of Green's function and the free space Green's functions can also be found in [16].

Like any other asymptotic method, it is important to highlight the validity domain in the first place. The total radiation is calculated assuming that the surface of the object is large enough so that the edges do not perturb the radiation of the elementary dipoles. Given this assumption, the diffracting object has to be large enough (a few wavelengths) and the grazing observation angles cannot be covered. The minimum observation distance is directly related to the accuracy of the asymptotic development of the Sommerfeld integral in the Green's functions expression. For not too low permittivities, the observation distance can be chosen from one wavelength of the diffracting object and given the working frequencies (a few gigahertz), reflected electric field can be calculated with a very good precision in any diffraction zone of the building going from very near-field of the building to its far-field.

\section{B. Model}

The presented Green's functions are associated with the interface between two semi-infinite media. A 2D model can naturally be used for building facades. The choice of semiinfinite Green's functions applied to building walls can be justified by the fact that at working frequencies (about $1 \mathrm{GHz}$ and beyond), the losses through the wall are large enough so that the wall be considered as a semi-infinite medium. However for windows with thin or multilayered glass parts which present very low loss, the semi-infinite assumption is no longer valid. In this case, we adopt a mathematical model [17] which allows applying the same type of Green's functions on stratified media by introducing an equivalent permittivity. In this way and for each incidence angle, the multilayered dielectric is replaced by a semi-infinite medium with an equivalent complex permittivity having the same reflection properties as the original medium. Obviously, the complex permittivity does not necessarily represent a physical medium and the solution is only valid in the reflection problem and not in the transmission one. Consequently, the building facade can 
eventually be described by a $2.5 \mathrm{D}$ model with large scale discontinuities.

In this paper, we consider $2 \mathrm{D}$ concrete-glass facades with variable physical and geometrical properties, so $3 \mathrm{D}$ geometries (e.g. balconies) are not treated. Sources of uncertainty in urban environment being numerous, we restrict the study to those coming from the architectural properties of the building; consequently we do not study the uncertainty on the source (e.g. radiating antennas) and the excitation is considered to be a plane wave. For the same reason, the direct path of the wave propagation, from the emitter to the receiver, or other indirect paths (e.g. ground reflection) is not taken into account because it does not involve buildings as reflecting objects.

\section{A few deterministic results}

Before integrating the stochastic approaches in the simulator, it is interesting to remind a few deterministic key results for the scattered field in the vicinity of the building. We consider the generic $12 \mathrm{~m} \times 12 \mathrm{~m}$ concrete-glass building profile presented in Fig. 1. The nominal parameter values are $\mathrm{W}=\mathrm{H}=1.25 \mathrm{~m}, \mathrm{D}_{1}=\mathrm{D}_{2}=0.6 \mathrm{~m}$ and $\mathrm{D}_{3}=\mathrm{D}_{4}=0.5 \mathrm{~m}$ while the relative concrete and glass permittivities are equal $\varepsilon_{\mathrm{rC}}=7$ and $\varepsilon_{\mathrm{rG}}=3.5$. Later in the statistical studies, we will see that these values present simply the mean of the uniformly distributed parameters.

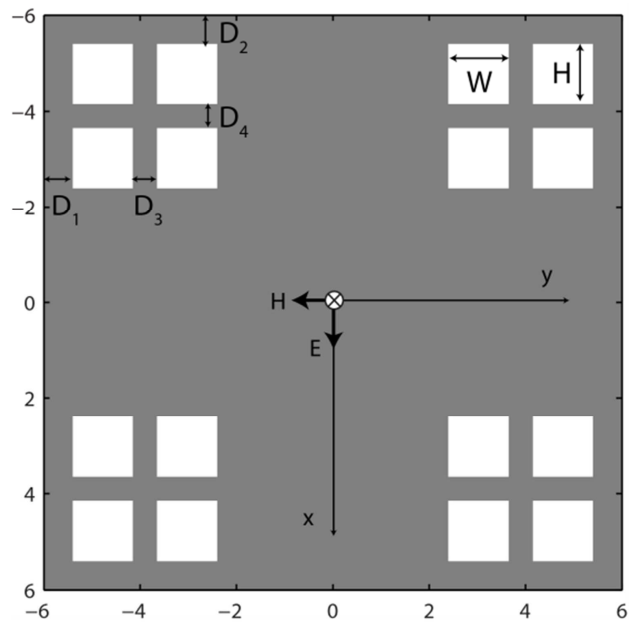

Fig. 1. Generic concrete-glass building facade presented

At first, the facade is illuminated by a TE polarized plane wave at $900 \mathrm{MHz}$ in normal $\left(\theta_{\mathrm{i}}=0^{\circ}\right)$ incidence and the reflected electric field is calculated in specular direction and in different diffraction zones of the building facade. The amplitude of the reflected electric field as a function of the observation distance is presented in Fig. 2. The observation distances can be referenced according to the definition of standard radiation/diffraction zones of a radiating/diffracting object. Fig. 2 also summarizes some of the most important existing definitions [18]-[21] where D represents the largest dimension of the building and $\lambda$ the free-space wavelength.

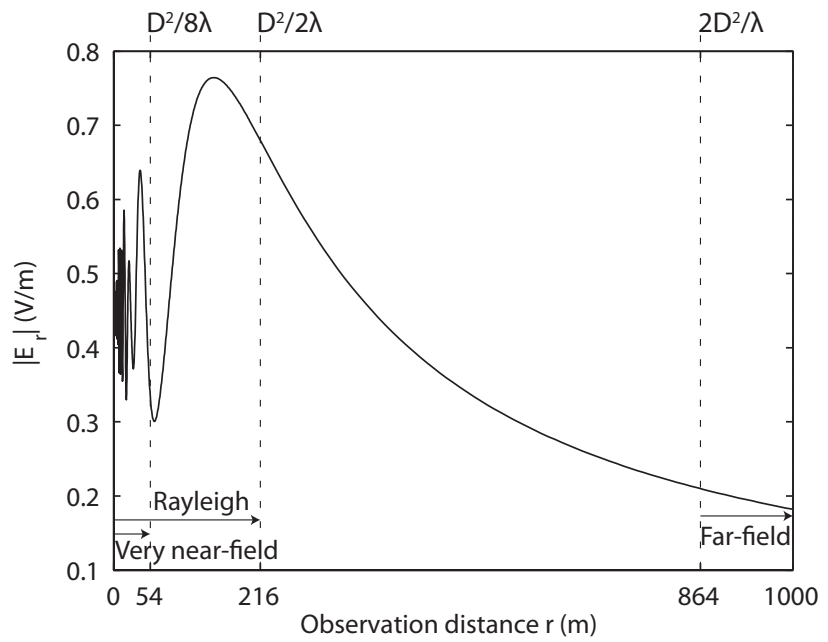

Fig. 2. Evolution of the amplitude of the reflected electric field in specular direction as a function of the observation distance from the generic building

As expected, in the very near-field of the building, the reflected electric field goes through a highly fluctuated zone. The variation becomes smoother further and the maximum radiation occurs before the end of the Rayleigh zone. After this peak, the amplitude decreases continuously through the intermediate zone (called Fresnel) into the far-field region.

The second key result shows the angular distribution of the electric field at a given distance. The plane wave is normally incident to the building facade in Fig. 1 at $900 \mathrm{MHz}$ and the scattered field is observed at $300 \mathrm{~m}$ from the building in Fresnel zone as a function of the observation angle. In Fig. 3, we notice that the scattered electric field presents a thin main lobe, given the large size of the building at this frequency $(36 \lambda)$. The field amplitude falls off rapidly so that after a few side lobes the received field becomes significantly low. Given the directivity of the building, the more grazing observation angles are thus not shown in this figure. Similar to the first result, the next section describes the influence of the input random parameters on the angular distribution of the electric field.

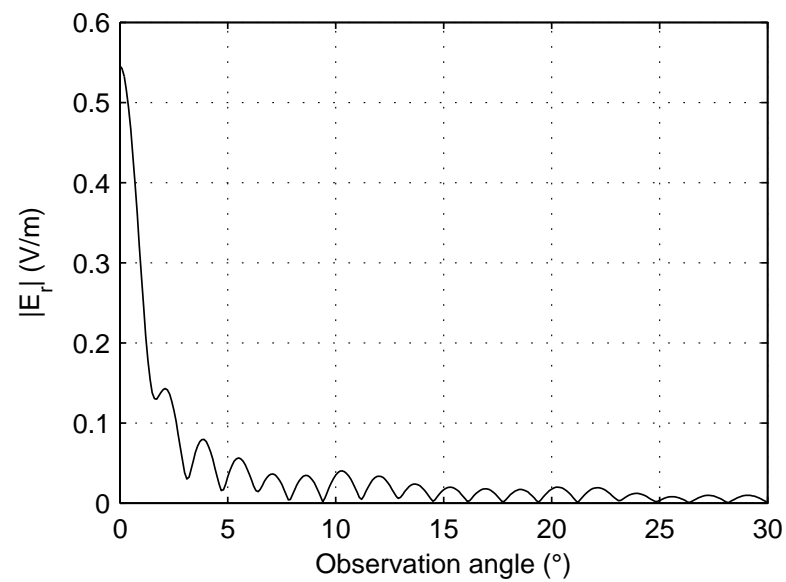

Fig. 3. Evolution of the amplitude of the reflected electric field at $300 \mathrm{~m}$ from the generic building and in non-specular directions 


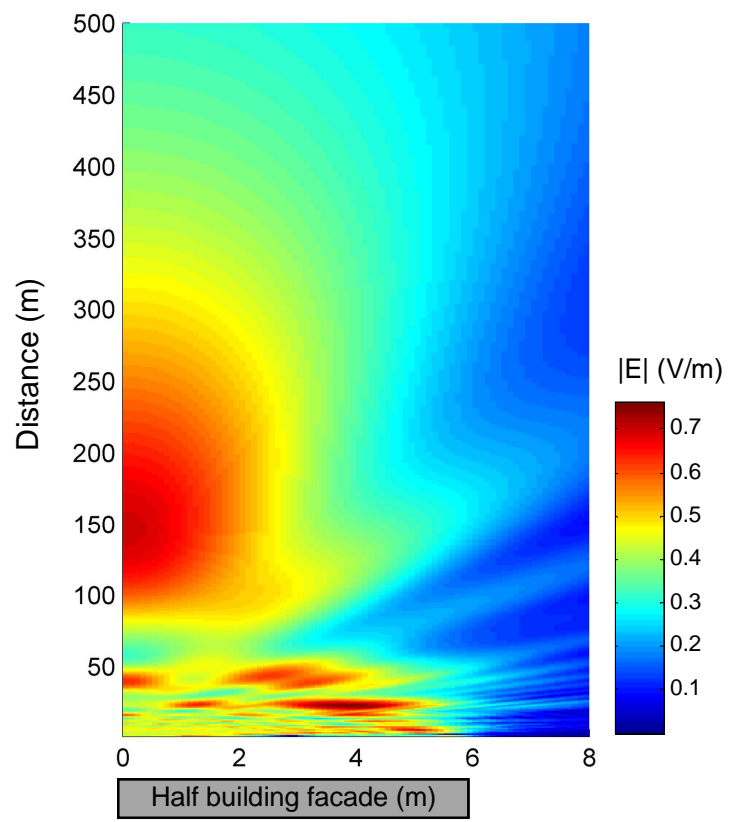

Fig. 4. Electric field overlay in the vicinity of the generic building and in the incidence plane

As a final result for the generic building profile, Fig. 4 presents the scattered field overlay in the incidence plane. The complexity of different diffraction zones of the building, in specular and non-specular directions, is very well observable in this figure. We will show in the next section how the uncertainty in the input parameters influence the amplitude of the scattered field and how the propagation of uncertainty can be studied, given the nature of the fluctuations in different diffraction zones. It is worth noting that for a given profile and with the computer used for our development, a single simulation with a fixed incidence and observation angle at a given distance takes approximately 3 seconds for a building discretization equal to $5 \mathrm{~mm}$.

\section{Statistical approach USing POLYNOMial ChaOS EXPANSION}

A first step in clarifying the complex mechanism between the inputs and the system response would be to underline the most influent parameters in a given configuration. In a very complex system with a large number of input random variables, this stage allows a smart elimination of the less influent parameters and thus a simplification of the whole process. Different types of multivariate sensitivity analysis can be performed [22]. It is important to note that some techniques provide the first order sensitivity indices where the influence of each input parameter on the system output is estimated. For many systems, this might prove to be sufficient but for some physical mechanisms the relation between the input and output reveals to be much more complicated, in this case a second or third order sensitivity analysis might be needed. This means that the separate impact of some input parameters is less important than their simultaneous effect. Parallel to the sensitivity analysis, some important statistical moments such as mean, variance, etc. can also be obtained. These results have a variable precision depending on the model, the sample size, the selected approach and the desired statistical moment. Finally in some cases, one can be interested in the total output distribution which is the most comprehensive representation of the process. Given a limited number of sample points, not all stochastic approaches can provide this amount of information concerning the system output including higher order statistical moments, rare events, etc. In this section, we present the polynomial chaos expansion which is a good candidate for a parsimonious able to provide with various types of statistical information.

Let us consider an input random vector $\boldsymbol{X}=\left[X_{1}, X_{2}, \ldots, X_{m}\right.$ ] and the random output related to the input parameters by a physical model $Y=\mathcal{M}(\boldsymbol{X})$. The polynomial chaos $(\mathrm{PC})$ is an advanced statistical method that gives a meta-model of the physical model by the polynomial expansion, provided that the input parameters are independent [23]:

$\boldsymbol{Y}=\sum_{\boldsymbol{\alpha} \in \mathbb{N}^{m}} a_{\boldsymbol{\alpha}} \psi_{\boldsymbol{\alpha}}(\boldsymbol{X})$

where $\boldsymbol{\alpha}=\left[\alpha_{1}, \ldots, \alpha_{M}\right]$ is the multi-index, $\psi_{\boldsymbol{\alpha}}$ are multivariate orthogonal polynomials and $a_{\boldsymbol{\alpha}}$ are unknown coefficients to be calculated. If the input random variables follow uniform distributions in the interval $[-1,1]$, the polynomials constituting the basis of the probabilistic space are of Legendre type [24]. In this paper, in order to calculate the unknown coefficients, the expansion is truncated keeping a given set of polynomials whose multi-indices belong to a subset $\mathcal{A} \subset \mathbb{N}^{m}$. Thus a regression problem is obtained:

$\boldsymbol{Y}=\sum_{\boldsymbol{\alpha} \in \mathcal{A}} a_{\alpha} \psi_{\alpha}(\boldsymbol{X})$

To solve this regression problem, the coefficients of the truncated expansion are estimated by minimizing the mean square error between the actual physical model computed at a given point of the experimental design and the truncated polynomial approximation of the latter equation, i.e. an ordinary least square resolution is employed. Let us consider the following experimental design of $N$ input points: $\boldsymbol{X}=$ $\left\{\boldsymbol{x}^{(1)} \ldots \boldsymbol{x}^{(N)}\right\}$, the coefficients of the truncated expansion are therefore approximated by the ordinary least-square estimator:

$\widehat{\boldsymbol{a}}=\left(\boldsymbol{\Psi}^{\mathrm{T}} \boldsymbol{\Psi}\right)^{-1} \boldsymbol{\Psi}^{\mathrm{T}} \mathbf{y}$

where $\widehat{\boldsymbol{a}}=\left\{\hat{a}_{\boldsymbol{\alpha}}\right\}_{\boldsymbol{\alpha} \in \mathcal{A}}^{T}$ is the vector of the estimated coefficients, $\boldsymbol{\Psi}=\left\{\psi_{\boldsymbol{\alpha}}\left(\boldsymbol{x}^{(1)}\right) \ldots \psi_{\boldsymbol{\alpha}}\left(\boldsymbol{x}^{(N)}\right)\right\}_{\boldsymbol{\alpha} \in \mathcal{A}}^{T}$ is the matrix of the polynomials computed at $\boldsymbol{X}$ and $\mathbf{y}=\left\{M\left(\boldsymbol{x}^{(1)}\right) \ldots M\left(\boldsymbol{x}^{(N)}\right)\right\}^{T}$ is the vector of the response of the physical model at $\boldsymbol{x}$. This regression solution would be less demanding in computational resources compared to the solution based on the projection of the expansion on the subspace associated to the coefficients of interest [25]. We bring to attention that this approach tends to build an experimental design in which the number of points will be limited by the conditioning of the least square 
information matrix and so by the number of polynomials kept in the truncation. Knowing that the truncation will certainly impact the quality of the meta-model, different types of truncation can be suggested. Besides, one needs to take on an iterative experimental design for which the number of points can be adjusted according to the desired quality of the metamodel. In this paper Latin Hypercube Sampling (LHS design) will be used and will be iteratively increased by Nested Latin Hypercube Sampling (NLHS) technique [23].

\section{A. Truncation}

In a full PC expansion, the infinite sum in equation (4) is reduced to a finite number $P$ of polynomials. The set $\left\{\psi_{\boldsymbol{\alpha}}, 0 \leq|\boldsymbol{\alpha}| \leq p\right\}$ represents all $\psi_{\boldsymbol{\alpha}}$ which are kept in the truncation where $p$ is the chosen maximal total degree of the retained polynomials. The number $P$ is related to the degree $p$ and the number $m$ of input variables by [25]:

$P=\left(\begin{array}{c}m+p \\ p\end{array}\right)$

If the dimension of the vector of input parameters increases and higher order polynomials are included, the full expansion meets a difficulty concerning its computational efficiency according to a large number of simulations in the experimental design. In this case, another type of truncation which results in a sparse polynomial chaos expansion seems to be more appropriate. In this second technique which is called the Least Angle Regression selection (LARS), only polynomials with the most significant impact on the model output are kept in the expansion [26], [27]. Thus this method provides a sparse representation of the physical model. The purpose is to iteratively select among a large full truncation the most significant polynomials depending on their correlation with the residual of the current meta-model. The used selection algorithm is detailed in [26]. As the most influent polynomials are added iteratively to the truncation, it provides a collection of possible truncation whose size also increases iteratively. The least-square estimation of the coefficients is therefore performed for each possible set to generate as many metamodels as possible while their quality needs to be assessed.

\section{B. Quality assessment}

In this paper, we use the so-called leave-one-out cross validation (LOOCV) technique [28]. Thereby, one point is taken out of the experimental design. The meta-model is constructed using the remaining points and the prediction error is calculated for the point which was set aside:

$$
\Delta^{(i)}=M\left(x^{(i)}\right)-\hat{M}^{(-i)}\left(x^{(i)}\right)
$$

where $x^{(i)}$ is the left-out point, $M\left(x^{(i)}\right)$ is the output value of the physical model for the point and $\hat{M}^{(-i)}$ is the meta-model obtained from the remaining points. The leave-one-out (LOO) error is calculated by repeating the procedure for each and every point in the experimental design.

$$
\operatorname{Err}_{\mathrm{LOO}}=\frac{1}{N} \sum_{i=1}^{N} \Delta^{(i)^{2}}
$$

where $N$ is the total number of points in the experimental design. Using this error, a determination coefficient can be derived [25]:

$$
\mathrm{Q}^{2}=1-\frac{\operatorname{Err}_{\mathrm{LOO}}}{\operatorname{Var}(Y)}
$$

where $\operatorname{Var}(Y)$ is the estimated variance of the output sample $Y$. The closer $\mathrm{Q}^{2}$ gets to one, the better is the quality of the metamodel in terms of generalization. The comparative study in [28] shows that overall, the LOOCV technique performs well in terms of generalization error bias. According to the desired $\mathrm{Q}^{2}$, the number and order of polynomials that are kept in the expansion will vary. If the LOOCV is used to assess the accuracy of the meta-model, it is also used to select the set of polynomials kept in the truncation providing the most accurate meta-model. In the LARS methodology described previously, the most influent polynomials are selected iteratively and added in the truncation. A LOOCV is therefore performed at each step of the LARS selection to assess the accuracy of the generated meta-models. In fact, because of a low number of points in the experimental design, the addition of two many LARS polynomials in the truncation could lead to an overfitting phenomenon. The number of LARS polynomials retained in the truncation is eventually the one that provides the higher $\mathrm{Q}^{2}$. Depending on the nature of physical process, this may result in a heavy calculation process.

\section{Global sensitivity and signature analysis}

Global sensitivity analysis aims at assessing the relative importance of each input parameter onto the output of the physical model. Sobol' indices [29] are widely used in this context. These sensitivity indices are usually computed by Monte Carlo simulation but from a polynomial chaos expansion, it is possible to compute global sensitivity indices of the model response related to the input parameters with a minimum computational effort [30], [31]. Let consider the PC expansion presented in (5), where $\mathcal{A} \subset \mathbb{N}^{m}$ represents the set of multi-indices $\boldsymbol{\alpha}$ corresponding to the polynomials kept in the truncation. The total Sobol' index to the input parameter $X_{\mathrm{i}}$ is then estimated by:

$\mathrm{S}_{\mathrm{i}}^{\mathrm{T}}=\frac{\sum_{\boldsymbol{\alpha} \in \mathcal{A}_{i}} a_{\boldsymbol{\alpha}}^{2}}{\sum_{\boldsymbol{\alpha} \in \mathcal{A} \backslash[\mathbf{0}]} a_{\boldsymbol{\alpha}}^{2}}$

where the denominator is the variance of the output, i.e. the sum of squares of all PC coefficients excluding $a_{\mathbf{0}}$ representing the mean. $\mathcal{A}_{\mathrm{i}}$ denotes the set of all multi-indices belonging to $\mathcal{A}$ with a non zero $i$-th component:

$A_{i}=\left\{\boldsymbol{\alpha} \in \mathcal{A}, \boldsymbol{\alpha}_{i} \neq 0\right\}$

In other words, the nominator represents the sum of squares of all PC coefficients for which the input parameter $X_{\mathrm{i}}$ is present in the expression of the multivariate polynomial.

Signature analysis is another way of assessing the relative importance of each input parameter in the output variance which is a direct result of the meta-model post processing. Considering centered and normalized polynomial, the variance 
of the meta-model is assessed in terms of each polynomial. To achieve such objective, one computes the relative magnitude of each coefficient. The relative magnitude of the coefficient $a_{\boldsymbol{\alpha}(i)}^{2}$ is defined as follow:

$\mathrm{s}_{\mathrm{i}}=\frac{a_{\boldsymbol{\alpha}(i)}^{2}}{\sum_{\boldsymbol{\alpha} \in \mathcal{A} \backslash[\mathbf{0}]} a_{\boldsymbol{\alpha}}^{2}}$

Where the denominator is the output variance as before and the numerator is simply the square of the coefficient of the $i^{\text {th }}$ polynomial in the expansion. In this way, the importance of each parameter is assessed through the amplitude of the polynomials involving that parameter. Yet the complexity of the phenomenon can be evaluated by the order and the amplitude of the higher-order polynomials. The nature of the interaction between parameters can be brought to light by looking into the relative order of the parameters composing the polynomial (which is not allowed by a crude sensitivity analysis). Thus a finer physical interpretation can be conducted from the polynomial-chaos meta-model.

\section{NUMERICAL RESULTS}

Although limited to architectural properties, several parameters can be identified whose variation may influence the scattered field and create an uncertainty on the calculated scattered field. In this study, we have chosen 8 random variables among all possible architectural variations. These parameters are defined for the generic $12 \mathrm{~m} \times 12 \mathrm{~m}$ concreteglass building illustrated in Fig. 1 .

In order to cover all possible values for different input parameters, no special hypothesis has been taken for the statistical distribution and all parameters are supposed to have a uniform variation over the authorized interval. The 8 parameters are as follows:

- Concrete relative permittivity: $U \varepsilon_{\mathrm{C}}=[5,9]$

- Glass relative permittivity: $\mathrm{U} \varepsilon_{\mathrm{G}}=[2,5]$

- Width of windows: $U_{\mathrm{W}}=[0.5,2] \mathrm{m}$

- Height of windows: $U_{H}=[0.5,2] \mathrm{m}$

- Horizontal distance of windows from the vertical edge of the building: $\mathrm{U}_{\mathrm{D} 1}=[0.2,1] \mathrm{m}$

- Vertical distance of windows from the horizontal edge of the building: $\mathrm{U}_{\mathrm{D} 2}=[0.2,1] \mathrm{m}$

- Horizontal distance between windows in each block: $\mathrm{U}_{\mathrm{D} 3}=[0,1] \mathrm{m}$

- Vertical distance between windows in each block: $\mathrm{U}_{\mathrm{D} 4}=[0,1] \mathrm{m}$

It is important to note that given the large variation intervals of the parameters, various building profiles can be generated, both from the geometrical and electrical point of view. The building in Fig. 1 is presented for the mean values of geometrical parameters; two extreme profiles are also shown in Fig. 5. In Fig. 5 (b), for the minimum value of parameters, the block of four windows in the corners are merged and the center of the facade is clear. In Fig. 5 (a), for the maximum value of parameters, four windows are merged in the center of the facade making a big bay window.

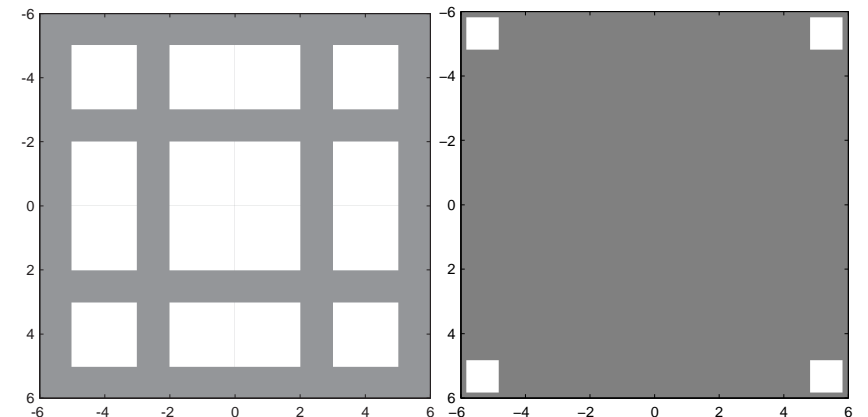

(a)

(b)

Fig. 5. Building profiles for (a) maximum (b) minimum values of geometrical parameters $\left(\mathrm{W}, \mathrm{H}, \mathrm{D}_{1}, \mathrm{D}_{2}, \mathrm{D}_{3}\right.$ and $\left.\mathrm{D}_{4}\right)$

The random variation of these parameters on one building (for example by a frequency change or due to an insufficient knowledge of the exact properties) or from one building to another, can cause large or small modifications in the wave propagation in the vicinity of buildings. As we will see, the impact would be more or less important according to different conditions such as wave polarization, incidence and observation angle or distance. As the scattered field behavior would be very different for each given condition, the latter parameters cannot be treated as random variables. In the following studies, we consider different scenarios in which the above parameters are fixed and the 8 random variables presented before change within their authorized intervals.

In this section, we describe two scenarios for the scattering assessment in the vicinity of the building. In both cases, a sufficient number of building profiles need to be generated. Here the sufficient number of samples is achieved when an acceptable determination coefficient $\left(Q^{2}\right)$ is obtained for the polynomial chaos meta-model built with LARS selection. This coefficient is directly related to the acceptable root mean square error for the phenomenon under consideration giving a more physical interpretation of the model quality.

\section{A. Observation distance}

Each random building profile is illuminated by a TE polarized plane wave at $900 \mathrm{MHz}$. The incidence and observation angles are fixed $\left(\theta_{\mathrm{i}}=\theta_{\mathrm{r}}=0^{\circ}\right)$. A few number of observation distances, from $50 \mathrm{~m}$ to $800 \mathrm{~m}$, representative of different diffraction zones, are selected to be studied.

The number of polynomials increases with the number of input parameters and the complexity of the model. Given the dramatic field fluctuations in the closest diffraction zone of the building, the chaos polynomial expansion becomes applicable only at the end of the very near-field region ( $54 \mathrm{~m}$ for a $12 \mathrm{~m}$ by $12 \mathrm{~m}$ building at $900 \mathrm{MHz}$ ). Indeed, below this limit, the high complexity of the output process results in a too low accuracy for a reasonable number of points. The first results can be obtained for the reflected field at $50 \mathrm{~m}$ from the building. The convergence of the PC expansion is verified for this worst case distance in Fig. 6. We can observe that an acceptable $Q^{2}=0.95$ can be obtained using 300 samples. For this distance, the total number of polynomials selected by LARS algorithm is $P=178$ from the total number of $P=(p+m) ! /(p ! m !)$ where $p=15$. This experimental design is 
kept for the other observation distances and guarantees the convergence of the technique for the further diffraction zones where the variation of the electric field is quite smooth. For a better comprehension, the Root Mean Square Error estimation associated to the value of $\mathrm{Q}^{2}=0.95$ obtained for 300 simulation points is equal to: $\mathrm{RMSE}_{\mathrm{LOO}}=7.3 \times 10^{-3} \mathrm{~V} / \mathrm{m}$.

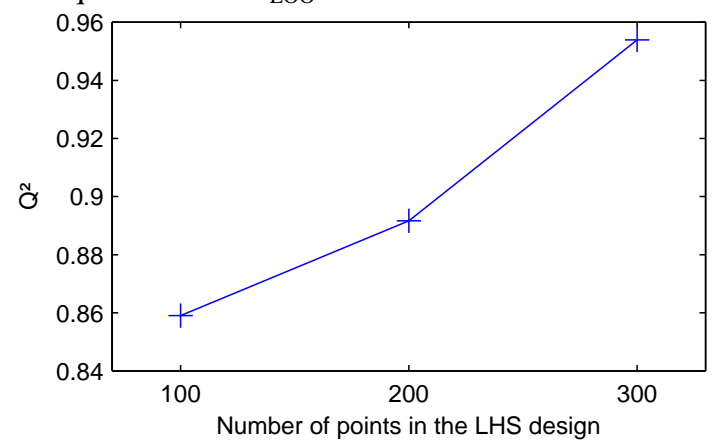

Fig. 6. Evolution of the determination coefficient as a function of the number of sample points in the LHS design calculated at $50 \mathrm{~m}$

Using this meta-model, the confidence intervals are calculated every 50 meters from the generic building facade. Fig. 7 shows the median and the distance between the 0.025 and 0.975 quantiles of the electric field superposed to the deterministic curve of Fig. 2 obtained by the mean values of the input architectural parameters. For a few distances the quantiles are compared to a 20000-sample Monte Carlo simulation. The medians indicated by a circle are very close to the mean values. The confidence interval decreases as the observation distance increases. This can be rather intuitive; for a far observer the field reflected from the building is a result of the influence of a set of physical and geometrical parameters attenuated by the distance which has the role of a predominant factor. This result shows the joint influence of all variables, yet we cannot distinguish which parameters are more influent in different region. A sensitivity analysis of the result can thus be useful.

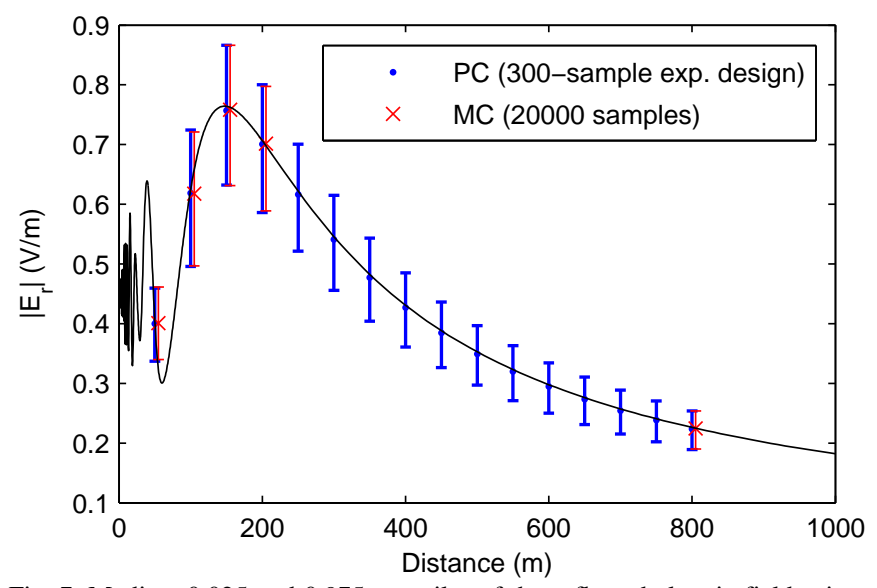

Fig. 7. Median, 0.025 and 0.975 quantiles of the reflected electric field using the meta-model constructed by the experimental design of 300 samples in different diffraction zones
As described in the previous section, the total Sobol' indices describe the importance of each input variable on the output variance. The total Sobol' indices are calculated for each input parameter and presented in Table I arranged in descending order. It appears that at $100 \mathrm{~m}$ the concrete permittivity is the input parameter that has the greatest influence on the output variance followed by the height and the width of the windows. The permittivity of glass appears far after these 3 variables. Other input parameters, involving the distribution of windows, do not have great influence. These results are in accordance with those of [14] where Sobol' indices were computed with perturbation technique.

Further details of the output variance expansion by computing the signature analysis of the expansion can now be presented. Fig. 8 makes a summary of the signature analysis for the most influent polynomials (only those which contribute for $95 \%$ of the output variance of the meta-model) at 100 meters from the building.

TABLE I

TOTAL SOBOL' INDICES FOR THE SPECULAR REFLECTION AT 100 M OF THE BUILDING FACADE

\begin{tabular}{|l|l|}
\hline Input parameter & Total Sobol' index \\
\hline $\mathrm{S} \varepsilon_{\mathrm{C}}$ & 0.5364 \\
$\mathrm{~S}_{\mathrm{H}}$ & 0.2094 \\
$\mathrm{~S}_{\mathrm{W}}$ & 0.2010 \\
$\mathrm{~S} \varepsilon_{\mathrm{G}}$ & 0.0906 \\
$\mathrm{~S}_{\mathrm{D} 1}$ & 0.0454 \\
$\mathrm{~S}_{\mathrm{D} 2}$ & 0.0363 \\
$\mathrm{~S}_{\mathrm{D} 4}$ & 0.0123 \\
$\mathrm{~S}_{\mathrm{D} 3}$ & 0.0075 \\
\hline
\end{tabular}

Fig. 8 presents the polynomials selected by LARS algorithm and arranged with regard to the LARS selection order. The values of the relative magnitude of the coefficients of the corresponding polynomials are presented by the height of color bars and can be read over the left axis. The order of the polynomials is indicated by the width of the bars and their contents (in terms of input parameters) by different colors introduced in the legend. Consequently the weight of each input parameter can be easily determined by the colors composing the bars. For instance, consider a polynomial of chaos selected at the 10th position by LARS algorithm, involving the concrete permittivity $\left(\varepsilon_{\mathrm{G}}\right)$ at order 1 and the width of windows $(\mathrm{W})$ at order 1 . The resulting multivariate polynomial of chaos will be the product of the univariate Legendre polynomial of order 1 involving $\varepsilon_{\mathrm{G}}$ and the univariate Legendre polynomial of order 1 involving $\mathrm{W}$. Thus the total order will be equal to 2 . This polynomial is shown in Fig. 8 by a bar of width 2 , composed of the two corresponding colors, each of which of width 1 . The relative magnitude of this polynomial is represented by the height of the bar and is equal to $6 \times 10^{-3}$. 


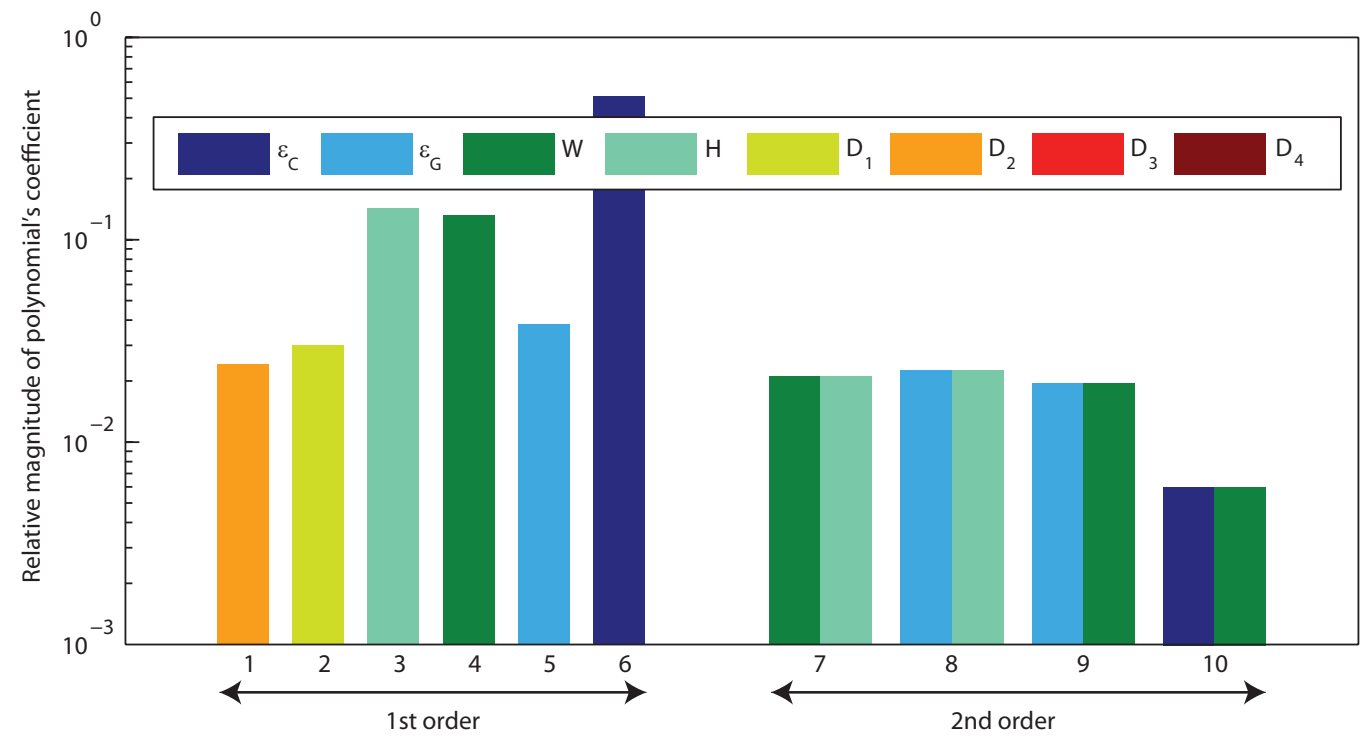

Most influent polynomials in the chaos expansion ( $95 \%$ of output variance) arranged by their selection order

Fig. 8. Signature analysis of the most influent polynomials in the LARS expansion associated to the order of polynomials and the weight of each input parameter calculated at $100 \mathrm{~m}$ from building facade

As we see, only 6 input parameters appear in the pure order category. The vertical and horizontal distances between windows $\left(D_{3}, D_{4}\right)$ are not part of the most important parameters which influence singly the reflected electric field form the building at this distance. Among the 6 present parameters, the concrete permittivity $\left(\varepsilon_{\mathrm{C}}\right)$ has the most important impact with an associated relative magnitude of 0.51 , followed by the dimensions of windows $(\mathrm{H}, \mathrm{W})$ with 0.14 and 0.13 relative magnitudes. As for the higher order polynomials, the interactions are limited to 2 parameters among the first 4 parameters $\left(\varepsilon_{\mathrm{C}}, \varepsilon_{\mathrm{G}}, \mathrm{W}, \mathrm{H}\right)$. All corresponding relative magnitudes are less important than the first order relative magnitudes, which means that the physical phenomenon has essentially a linear behavior with respect to the 6 first variables. We also point out that beyond this linear aspect, the most influent polynomials on output variance are the interaction polynomials and none of the pure order polynomials of level 2 appears in the list. Knowing that the reflected field behaves differently in different diffraction zones, the most influent parameters vary according to the distance. The relative magnitudes should thus be performed for different distances.

With the best meta-model available as a polynomial expansion, we can easily obtain the statistical distribution of the reflected field at different distances. The probability density functions for a few observation distances are traced in Fig. 9. Given the quality of the model (Fig. 6), we expect that these distributions be very close to the reality. The results are compared to an enriched Monte Carlo simulation with 20000 samples in the LHS design. It is important to remind that the PDF obtained by PC is a result of a meta-model constructed with 300 samples in the experimental design. As can be observed in Fig. 9, the distributions obtained from the PC expansions are almost exactly equal to the reference ones obtained by MCs. Note that the standard deviation is

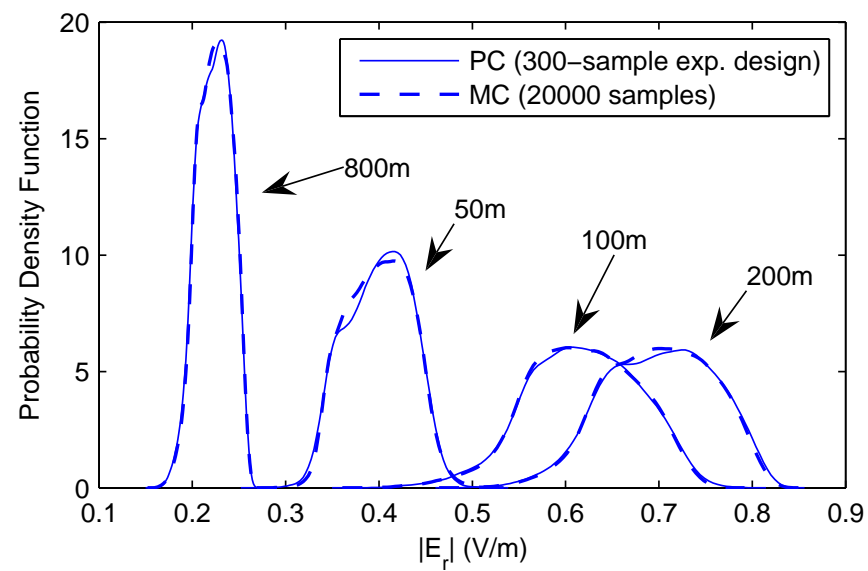

Fig. 9. Statistical distribution of the amplitude of the reflected electric field for a few number of observation distances obtained by Polynomial Chaos (PC) and Monte Carlo (MC)

In order to highlight the slow convergence of the Monte Carlo method, the PC results are compared to that of a poor MC simulation. For two distances ( $r=50 \mathrm{~m}$ and $\mathrm{r}=800 \mathrm{~m}$ ), the PC probability density functions are compared to two different runs for a 300-sample crude Monte Carlo in Fig. 10. We can observe that the pdf changes considerably between the first and the second run. For each different 300-run, we would obtain a quite different result among which we can eventually find a result which is close to the result predicted by PCE. We can conclude that the statistical moments do not converge for 300 samples. In the Monte Carlo method, the higher the moment, the slower would be the convergence as a function of sample number. While an acceptable accuracy may be obtained for mean, whole distributions, especially the tails are far from being precise using 300 samples. In other words, the variance of the 300 -sample estimators is not negligible and cannot be represented by the results of a single run. 


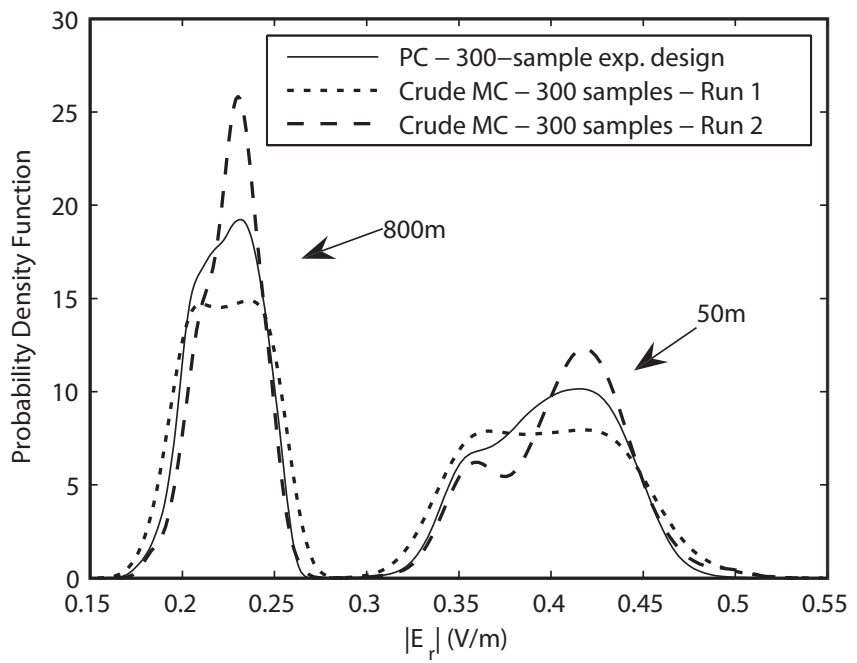

Fig. 10. Probability density functions obtained by Polynomial Chaos (PC) and two different runs of crude Monte Carlo (MC) with 300 sample points

\section{B. Observation angle}

Each random building profile is illuminated by a TE polarized plane wave at $900 \mathrm{MHz}$ and in normal incidence $\left(\theta_{\mathrm{i}}=0^{\circ}\right)$. The angular distribution of the electric field is studied at a fixed observation distance.

The radiation pattern of the building facade is highly directive and the field level for the side lobes decreases rapidly (Fig. 3). We can fix a deviation limit from the specular direction $\left(\theta_{\mathrm{r}}=0^{\circ}\right)$ beyond which the field level is negligible. According to the distribution, $\theta_{\mathrm{r}}=10^{\circ}$ (in zy plane of Fig. 1) can be a reasonable limit in this study. As the field goes through different local maxima and minima and as the level is globally low, non-specular reflections present a challenge for the polynomial chaos expansion. For $10^{\circ}$, the evolution of the leave-one-out $\mathrm{Q}^{2}$ as a function of the number of samples in the experimental design is shown in Fig. 11. For the same metamodel quality $\mathrm{Q}^{2}=0.95$ as the previous scenario, we need a larger sample size $\mathrm{N}=800$. We note that the Root Mean Square Error estimation associated to this $\mathrm{Q}$ value is equal $2.4 \times 10^{-3} \mathrm{~V} / \mathrm{m}$. The total number of polynomials selected by LARS algorithm in this case is $P=434$. For a few observation angles, the confidence intervals are calculated using the 800 points in the LHS design and are superposed in Fig. 12 to the angular distribution of electric field obtained by the mean values of the input parameters of the building facade.

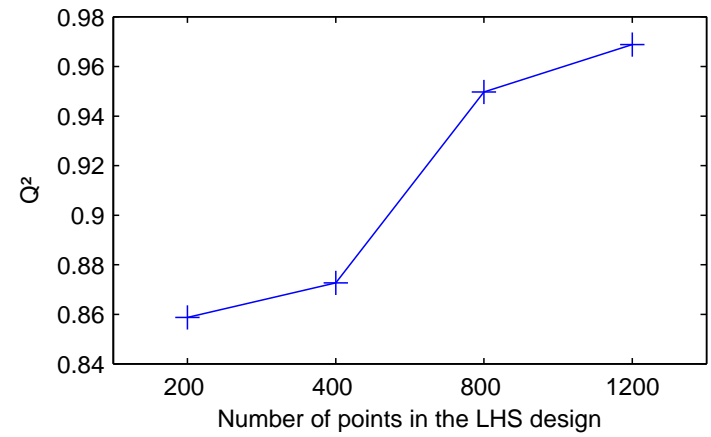

Fig. 11. Evolution of the determination coefficient as a function of the number of sample points in the LHS design calculated at $100 \mathrm{~m}$ for $\theta_{\mathrm{r}}=10^{\circ}$

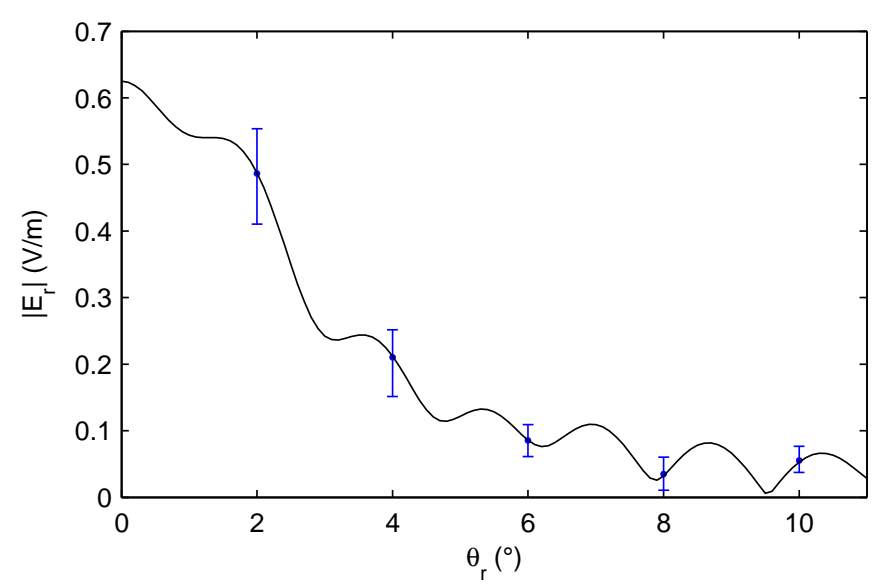

Fig. 12. Median, 0.025 and 0.975 quantiles of the reflected electric field using the experimental design of 800 samples at $100 \mathrm{~m}$ for different observation angles

In order to complete the information conveyed by the confidence intervals, signature and relative magnitude analyses can be added to identify the most important parameters. A similar illustration to the previous scenario is presented in Table II and Fig. 13 for the largest studied observation angle of $\left(\theta \mathrm{r}=10^{\circ}\right)$ at $100 \mathrm{~m}$ from the building facade. In Table II are presented the total Sobol' indices for this case. The relative importance of the input parameters is not the same as the previous study. Particularly variables involving lateral modifications in the geometry (W, D1 and D3) become clearly more important than the previous study. TABLE II

TOTAL SOBOL' INDICES FOR THE NON SPECULAR REFLECTION $\left(10^{\circ}\right)$ AT $100 \mathrm{M}$ OF THE BUILDING FACADE

\begin{tabular}{|l|l|}
\hline Input parameter & Total Sobol' index \\
\hline $\mathrm{S} \varepsilon_{\mathrm{C}}$ & 0.3415 \\
$\mathrm{~S}_{\mathrm{D} 1}$ & 0.3031 \\
$\mathrm{~S}_{\mathrm{W}}$ & 0.2950 \\
$\mathrm{~S} \varepsilon_{\mathrm{G}}$ & 0.2346 \\
$\mathrm{~S}_{\mathrm{D} 3}$ & 0.1758 \\
$\mathrm{~S}_{\mathrm{H}}$ & 0.1309 \\
$\mathrm{~S}_{\mathrm{D} 4}$ & 0.0807 \\
$\mathrm{~S}_{\mathrm{D} 2}$ & 0.0611 \\
\hline
\end{tabular}

Concerning the signature analysis presented in Fig. 13, the interpretation is much more complicated than the previous study. In order to simplify the readability of the graph, we deliberately fixed the importance of the polynomials to $80 \%$ of the output variance. For instance, consider a polynomial of chaos selected at 15 th position by LARS algorithm, involving the width of windows (W) at order 2 and the height of windows $(\mathrm{H})$ at order 1 . The resulting multivariate polynomial of chaos will be the product of the univariate Legendre polynomial of order 2 involving $\mathrm{W}$ and the univariate Legendre polynomial of order 1 involving $\mathrm{H}$. Thus the total order will be equal to 3 and the polynomial will be represented at 15 th position in this figure by a bar of width 3 and composed of the two corresponding colors. The relative magnitude of this polynomial is represented by the bar's height and is equal to $15 \times 10-3$. 


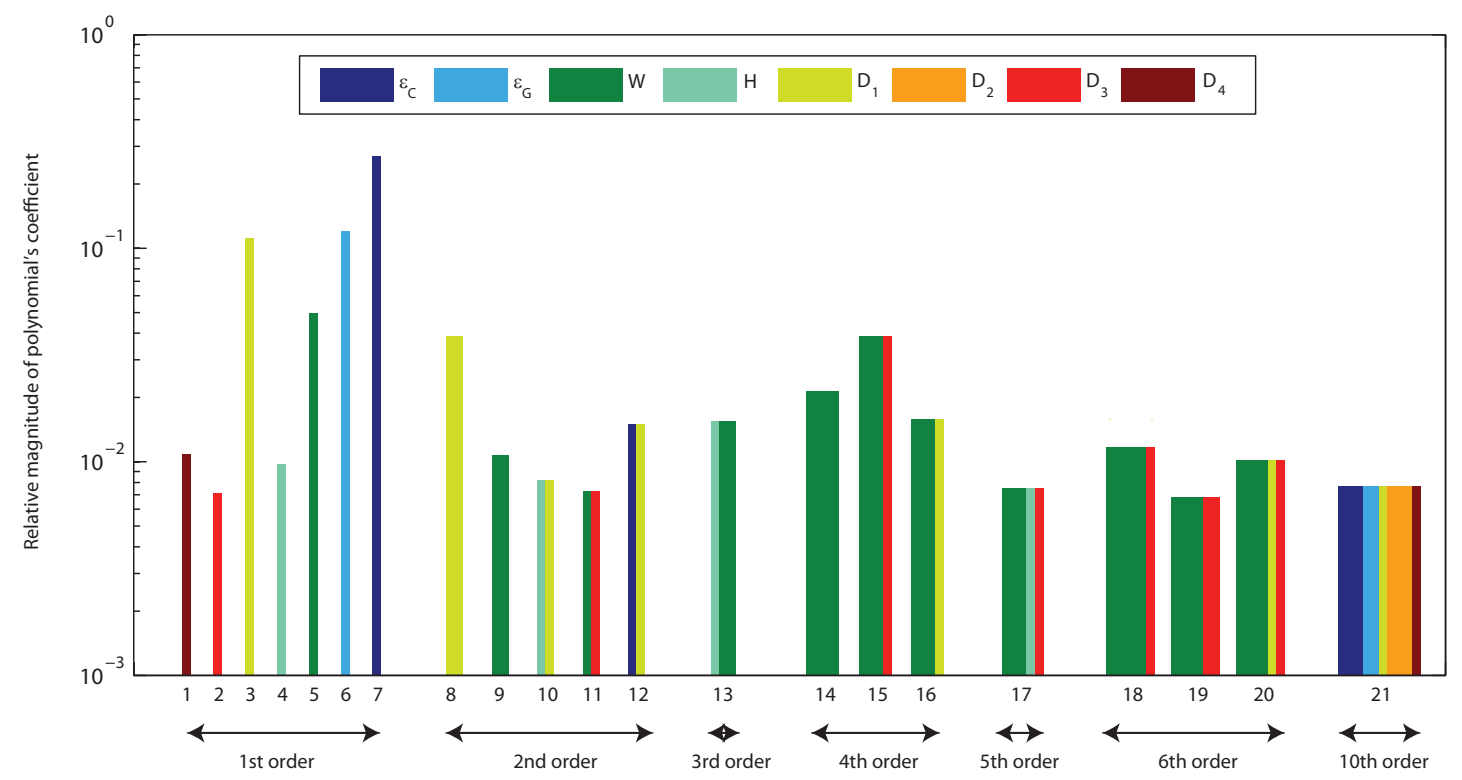

Most influent polynomials in the chaos expansion ( $80 \%$ of output variance) arranged by their selection order

Fig. 13. Signature analysis of the most influent polynomials in the LARS expansion associated to the order of polynomials and the weight of each input parameter calculated for $\theta_{\mathrm{r}}=10^{\circ}$ at $100 \mathrm{~m}$ from building facade

We observe that in non-specular reflection, the global level of sensitivity indices is lower than the specular reflection while the order of the most influent polynomials varies from a pure to the 6th order. All of the input parameters except for D2 appear in the pure order category among which the concrete and glass permittivities $(\varepsilon \mathrm{C}, \varepsilon \mathrm{G})$ occupy the first ranks followed by the lateral distance of windows from the edge of the building (D1) and the width of the windows (W). Both of parameters represent horizontal distances. Given the deviation of the observation point from the specular direction and the wave polarization, the influence of horizontal distances and dimensions is obviously more important than that of a vertical distance or dimension. As far as higher order polynomials are concerned, we note a strong presence of the width of the windows $(\mathrm{W})$ in different interactions and a rare presence of the vertical distances (D2 and D4). Although the level of each sensitivity index of higher order polynomial is low when considered separately, some of them are of the same order as that of the pure order polynomials. It is important to note that if in this case we display the polynomials participating up to $95 \%$ of output variance, as in the previous scenario, the figure would be much more loaded with higher order polynomials up to order 15. As mentioned previously, for the sake of comparison between the model qualities in the two scenarios, we built the second model using 800 samples. Given the low amplitude of electric field in the non-specular case, we examined the validity of results by 1200 samples which results in a higher Q2 (Fig. 11). No fundamental differences have been observed except that the two Sobol' indices $\mathrm{S} \varepsilon \mathrm{C}$ and $\mathrm{SW}$ were interchanged in Table II and the signature analysis of Fig. 13 stops at 6th order polynomials. Since the first three sensitivity indices are of the same order, the conclusions remain the same.

The statistical distribution of the reflected electric field at $100 \mathrm{~m}$ from the building is presented for different observation angles in Fig. 14 and compared to a 20000-sample Monte Carlo simulation. As expected, the probability density function for large non-specular angles $\left(6^{\circ}\right.$ and $\left.10^{\circ}\right)$ is very narrow resulting in low dispersion. Given the very small value of the electric field for these angles, no significant information can be obtained from the field distribution. For $\theta_{\mathrm{r}}=2^{\circ}$, the distribution is similar to the specular reflection and the signature analysis is less complex than those of large reflection angles. In this case, the polynomials contributing up to $95 \%$ of output variance are of pure order and first order interaction. The more the observation angle deviates from the specular direction, the larger gets the order of most important polynomials. Given the wave polarization and the observation plane (zy), this will more involve the horizontal geometrical parameters $\mathrm{W}, \mathrm{D}_{3}$ and $\mathrm{D}_{1}$ compared to the specular reflection.

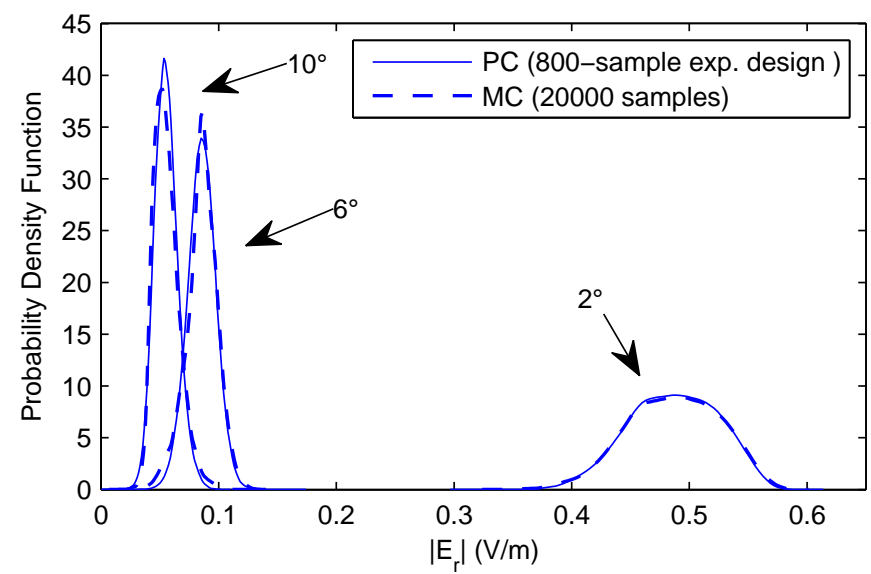

Fig. 14. Statistical distribution of the amplitude of the reflected electric field for a few number of observation angles obtained by PC 


\section{CONCLUSION}

In this paper we have implemented the polynomial chaos expansion in order to estimate the influence of uncertainties in geometrical and physical properties of a building facade on the scattered electric field. This technique allows an efficient multivariate sensitivity analysis for a complex problem. The number of chosen input random variables is 8 and using this technique, the number of required input samples has been reduced by more than one order of magnitude compared to a Monte Carlo approach for the same precision in output distribution. Presented confidence intervals of the scattered field show considerable variation in different scenarios and different diffraction zones. By adding a statistical dimension to a deterministic calculation and using the techniques of the propagation of uncertainties in a complex scenario, field distribution as an output of a wave propagation simulator can be presented by a confidence interval making the result more meaningful for subsequent decision making.

\section{REFERENCES}

[1] M. F. Iskander, and Z. Yun, "Propagation prediction models for wireless communication systems," IEEE Trans. Antennas Propagat., vol. 50, pp. 662-673, March 2002.

[2] Y. Okumura, E. Ohmori, T. Kawano, and K. Fukuda, "Field strength variability in VHF and UHF land mobile service," Rev. Elect. Comm. Lab., vol. 16, pp. 825-873, Sept.-Oct. 1968.

[3] G. L. Turin, F. F. Clapp, T. L. Johnston, S. B. Fine, and D. Lavry, "A statistical model of urban multipath propagation," IEEE Trans. Veh. Technol., vol. 21, pp. 1-9, Feb. 1972.

[4] P. Pongsilamanee, and H. L. Bertoni, "Specular and nonspecular scattering from building facades," IEEE Trans. Antennas Propagat., vol. 52, pp. 1879-1889, July 2004.

[5] S. Y. Seidel, and T. S. Rappaport, "Site-specific propagation prediction for wireless in-building personal communication system design," IEEE Trans. Veh. Technol., vol. 43, pp.879-891, Nov. 1994.

[6] D. Xiu, "Fast Numerical Methods for Stochastic Computation: A review," Communications in Computational Physics, Vol. 5, pp. 242272, Feb. 2009.

[7] B. Sudret, Uncertainty propagation and sensitivity analysis in mechanical models - Contributions to structural reliability and stochastic spectral methods, Habilitation à diriger des recherches, Université Blaise Pascal, Clermont-Ferrand, France, 2007.

[8] C. Chauvière, J. S. Hesthaven and L. C. Wilcox, "Efficient Computation of RCS From Scatterers of Uncertain Shapes," IEEE Transactions on Antennas and Propagation, vol. 55, pp. 1437-1448, May 2007.

[9] J. Silly-Carette, D. Lautru, A. Gati, M.F. Wong, J. Wiart and V.F. Hanna, "Determination of the uncertainty on the specific absorption rate using the stochastic collocation method and the FDTD," IEEE Antennas and Propagation Society International Symposium (APS), pp. 1-4, 2008.

[10] P. Bonnet, F. Diouf, C. Chauvière, S. Lalléchère, M. Fogli and F. Paladian, "Numerical simulation of a Reverberation Chamber with a stochastic collocation method," Compte Rendus Physique, vol. 10, pp. 54-64, 2009.

[11] R. S. Edwards, A. C. Marvin, S. J. Porter, "Uncertainty Analyses in the Finite-Difference Time-Domain Method," IEEE Transactions on Electromagnetic Compatibility, vol. 52, pp. 155-163, Feb. 2010.

[12] A. Ghanmi, Y. Pinto, A. Hadjem, E. Conil, J. Wiart and O. Picon, "Uncertainty propagation in the SAR induced in the head using polynomial chaos decomposition," Mediterranean Microwave Symposium (MMS), pp. 73-76, 2011.

[13] P. Manfredi, D. V. Ginste, D. De Zutter, F. G. Canavero, "Uncertainty Assessment of Lossy and Dispersive Lines in SPICE-Type Environments," IEEE Transactions on Components, Packaging and Manufacturing Technology, vol.3, pp. 1252-1258, July 2013.

[14] S. Mostarshedi, B. Sudret, E. Richalot, J. Wiart and O. Picon, "Multivariate uncertainty analysis of scattered electric field from building facades in urban environment," AES, 16-19 April 2012, Paris.
[15] R. E. Calfisch, "Monte Carlo and quasi Monte Carlo methods," Acta Numerica, Cambridge University Press, pp. 1-49, 1998.

[16] S. Mostarshedi, E. Richalot, J.-M. Laheurte, M.-F. Wong, J. Wiart, O. Picon, "Fast and accurate calculation of scattered electromagnetic fields from building faces using Green's functions of semi-infinite medium," IET Microwaves, Antennas \& Propagation, vol.4, pp. 72-82, Jan. 2010.

[17] S. Mostarshedi, E. Richalot and O. Picon, "Semi-infinite reflection model of a multilayered dielectric through equivalent permittivity calculation," Microwave and Optical Technology Letters, vol. 51, pp. 290-294, Feb. 2009.

[18] IEEE standard definitions of terms for antennas, IEEE Trans. Antennas Propagat., vol. 17, pp. 262-269, May 1969.

[19] A. Yaghjian, "An overview of near-field antenna measurements," IEEE Trans. Antennas Propagat., vol. 34, pp. 30-45, Jan. 1986.

[20] S. Laybros, P. F. Combes, H. J. Mametsa, "The very-near-field region of equiphase radiating apertures," IEEE Antennas and Propagat. Magazine, vol. 47, pp. 50-66, Aug. 2005.

[21] S. Laybros, P. F. Combes, "On radiating-zone boundaries of short $\lambda / 2$, and $\lambda$ dipoles," IEEE Antennas and Propagat. Magazine, vol. 46, pp. 53-64, Oct. 2004.

[22] B. Iooss, "Revue sur l'analyse de sensibilité globale de modèles numériques," Journal de la Société Française de Statistique, vol. 152, pp. 3-25, Jan. 2011.

[23] G. Blatman and B. Sudret, "An adaptive algorithm to build up sparse polynomial chaos expansion for stochastic finite element analysis," Probabilistic Engineering Mechanics, vol. 25, pp. 183-197, April 2010.

[24] D. Xiu and G. Karniadakis, "The wiener-askey polynomial chaos for stochastic differential equation," SIAM Journal on Scientific Computing, vol. 24, no. 2, pp. 619-644, 2002.

[25] G. Blatman and B. Sudret, "Sparse Polynomial Chaos expansion and adaptative stochastic finite elements using a regression approach," Comptes Rendus Mécaniques, vol. 336, pp. 518-523, June 2008.

[26] G. Blatman and B. Sudret, "Adaptive sparse Polynomial Chaos expansion based on least angle regression," Journal of Computational Physics, vol. 230, pp. 2345-2367, March 2011.

[27] B. Efron, T. Hastie, I. Johnstone and R. Tibshirani, "Least Angle Regression," The Annals of Statistics, vol. 32, no. 2, pp. 407-499, 2004.

[28] A.M. Molinaro, R. Simon and R.M. Pfeiffer, "Prediction error estimation: a comparison of resampling methods," Bioinformatics, vol. 21, no. 15, pp. 3301-3307, 2005.

[29] I.M. Sobol", "Global sensitivity indices for nonlinear mathematical models and their Monte Carlo estimates", Mathematics and Computers in Simulation, vol.55, pp. 271-280, 2001.

[30] B. Sudret, "Global Sensitivity Analysis using Polynomial Chaos Expansion," Reliability Engineering \& System Safety, vol. 93, pp. 964979, July 2008.

[31] G. Blatman and B. Sudret, "Efficient computation of global sensitivity indices using sparse polynomial chaos expansions, " Reliability Engineering \& System Safety, vol. 95, pp. 1216-1229, Nov. 2010. 